\title{
Case Report \\ Hallervorden-Spatz Syndrome: Case Report of a Typical Form
}

\author{
Leandro de Holanda da Rocha', Milena Nunes Alves de Sousa ${ }^{1 *}$, Paulo Roberto Veiga Quemelo² and Paulo Antônio Farias Lucena' \\ 'Centro Universitário de Patos, Brazil \\ ${ }^{2}$ Centro Universitário São Camilo, Brazil
}

\begin{abstract}
Hallervorden-Spatz syndrome is a rare neurodegenerative disease, related to mutations in a gene located on chromosome 20p13. Hallervorden-Spatz syndrome is characterized by iron accumulation in the basal ganglia, which leads to variable neurologic manifestations. It is reported the case of a 6 years old male patient, with history of neuro psycho motor development involution noticed since 1 year and 5 months of age and progressive development of dystonia, mostly on upper limbs and neck. Brain Magnetic Resonance Imaging (MRI) revealed bilaterally symmetric signal changes in globus pallidus and in the posterior limb of the internal capsule, findings that suggest neurodegenerative disease with iron accumulation or metabolic disease.
\end{abstract}

Keywords: Hallervorden-Spatz syndrome; Neurodegeneration; Iron accumulation; Basal ganglia

\section{Introduction}

Hallervorden-Spatz syndrome is a rare neurodegenerative condition (prevalence 1-9/1000000) related to mutations in a gene located on chromosome 20p13. First described in 1922 by two German physicians, it is characterized by an accumulation of iron in the basal nuclei, with consequent variable neurological manifestations [1-3]. The mutation occurs in the gene producing the enzyme pantothenic nattokinase 2, which results in an inborn error of coenzyme A metabolism, and the disease may also be called pantothenate kinaseassociated neurode generation. Despite this, the pathophysiology is still unknown $[1,4]$.

There are two forms of the syndrome presentation: typical and atypical. Typically, the clinical picture starts early, usually in early childhood. The symptoms are intense, with rapid evolution, and the main clinical findings are ataxia, postural difficulties, and extra pyramidal symptoms, such as dystonia, chorea and Parkinsonism [5]. The atypical is more heterogeneous, having a late onset and slower evolution. Psychiatric manifestations (depression, impulsiveness and aggressiveness) and speech disorders are more prominent, with less accentuated motor involvement [6].

Clinical data associated with the "tiger's eye" sign on MRI contribute to the diagnosis of Hallervorden-Spatz syndrome. Treatment is symptomatic and multidisciplinary [7].

Citation: da Rocha LH, de Sousa MNA, Quemelo PRV, Lucena PAF. Hallervorden-Spatz Syndrome: Case Report of a Typical Form. Ann Clin Case Stud. 2021; 3(2): 1045.

Copyright: $\odot 2021$ Leandro de Holanda da Rocha

Publisher Name: Medtext Publications LLC

Manuscript compiled: Dec $03^{\text {rd }}, 2021$

*Corresponding author: Milena Nunes Alves de Sousa, Centro Universitário de Patos, Paraiba, Brazil, E-mail: milenanunes@ fiponline.edu.br

\section{Case Presentation}

Male child, six years old, with history of neuropsychomotor involution from one year and five months onwards and progressive development of dystonia, predominantly in the upper limb sand neck.

Family history of consanguineous parents and HallervordenSpatz syndrome in a brother, paternal aunt, maternal second and third cousins. All family members from consanguineous marriages and with premature deaths until the second decade of life.

During physical examination, he presented rapid arrhythmic involuntary movements, unbalanced gait, parkinsonism and dystonia in the left upper limb and neck. Dysarthria was observed, but the patient understood simple questions.

Brain MRI showed bilaterally symmetrical signal changes in the globus pallidus and in the posterior arm of the internal capsule, characterized by hyposignalon T1 weighting and hypersignal on T2 weighting. Findings suggest neurodegenerative disease due to iron deposition or metabolic disease (Figure 1).

Upon tracking of anatomical alterations by SNP-array, the patient had 11 homozygous genomic segments, including on chromosome 20 p13, indicating that the parents have a common ancestor, which is in agreement with the consanguinity reported in the anamnesis.

The child uses botulinum toxin every six months, with a moderate reduction in dystonia only in the first months after application. He also uses trihexyphenidyl, with a partial effect on Parkinsonism and extra pyramidal disorders. Therefore, the treatment resulted in a decrease in the clinical symptoms, but it did not prevent the progression of the disease.

\section{Discussion}

The disease is a clinical-radiological entity characterized by neurodegeneration with relevant extra pyramidal symptoms, intellectual impairment and iron accumulation in the brain identified by MRI [5].

The tiger eye sign on MRI, revealed by bilateral hypointensity of 


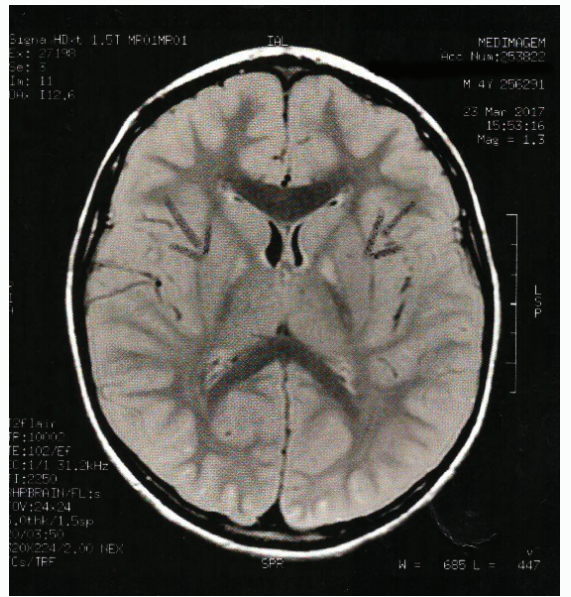

Figure 1: Axial MRI cut revealing "tiger eye sign".

the globus pallidus with a central area of hyper intensity on T2, is characteristic of the pathology and helps to differentiate from other neuro degenerations associated with brain iron accumulation. The genetic test pointing out alterations in chromosome 20 also helps to elucidate the case $[2,6]$.

In the symptomatic treatment of this syndrome, dopaminergic agents can be used for tremors, botulinum toxin and baclofen for dystonia, and benzodiazepines for chorea. When dopaminergic agents are ineffective, anticholinergics can be used. Systemic iron chelators, such as deferoxamine, have been used, but without any benefits. Research on the administration of coenzyme A and high doses of pantothenate is still ongoing $[1,7,8]$.

\section{References}

1. Bokhari MR, Zulfiqar H, Bokhari SRA. Hallervorden Spatz Disease. Stat Pearls. 2021.

2. Pawar Y, Kalra G, Sonavane S, Shah N. A case of Hallervorden-Spatz disease presenting as catatonic schizophrenia. Indian J Psychiatry. 2013;55(4):386-9.

3. Alami B, Tizniti S. [Hallervorden-Spatz disease in a young adult]. Pan Afr Med J. 2014;19:62.

4. Arber CE, Li A, Houlden H, Wray S. Review: Insights into molecular mechanisms of disease in neurodegeneration with brain iron accumulation: unifying theories. Neuropathol Appl Neurobiol. 2016;42(3):220-41.

5. Tonekaboni SH, Mollamohammadi M. Neurodegeneration with Brain Iron Accumulation: An Overview. Iran J Child Neruol. 2014;8(4):1-8.

6. Nassif D, Pereira JS, Spitz M, Capitão C, Faria A. Neurodegeneration with brain iron accumulation: A case report. Dement Neuropsychol. 2016;10(2):160-4.

7. Dashti M, Chitsaz A. Hallervorden-Spatz disease. Adv Biomed Res. 2014;3:191.

8. Arber CE, Li A, Houlden H, Wray S. Review: Insights into molecular mechanisms of disease in neurodegeneration with brain iron accumulation: unifying theories. Neuropathol Appl Neurobiol. 2016;42(3):220-41. 